\title{
Situación actual de la convergencia europea de los estudios de Ciencias de la Salud
}

\author{
Competencias genéricas. ¿Quien las define?, \\ ¿Cómo se adquieren?, ¿Cómo se evaluan? \\ J. Carreras Barnés \\ Departament de Ciències Fisiològiques I. \\ Facultat de Medicina. Universitat de Barcelona
}

La adopción de un sistema de titulaciones fácilmente comparables y comprensibles, señalada ya en la Declaración de Bolonia de 1999 como uno de los objetivos de la construcción del Espacio Europeo de Educación Superior, requiere la definición de los perfiles profesionales propios de cada titulación y la determinación de los correspondientes resultados de aprendizaje en términos de competencias; entendidas éstas como combinaciones dinámicas de los atributos (con respecto al conocimiento y a su aplicación, a las actitudes y las responsabilidades) que describen los resultados. La definición de competencias permite flexibilidad y autonomía en la construcción de los planes de estudios; a la vez que facilita el establecimiento de objetivos transparentes y dinámicos para los programas de formación, la adopción de métodos de docencia/aprendizaje más centrados en el estudiante, el desarrollo de sistemas de evaluación de los resultados más adecuados y la formulación de indicadores de nivel que puedan ser comprendidos internacionalmente. Además, tiene la ventaja adicional de que constituye un lenguaje comprensible tanto para las instituciones académicas como para los organismos profesionales y para otros grupos sociales interesados en la educación.

Resulta, pues, evidente la necesidad de definir los catálogos de competencias correspondientes a los perfiles profesionales de las diversas titulaciones universitarias; tanto aquellas que son propias de determinada titulación (competencias específicas) como aquellas que son comunes a todas o a la gran mayoría de titulaciones (competencias genéricas o transversales). Ahora bien, ello no puede ser el simple resultado de la reflexión de la institución académica responsables de la titulación. El cambio y la variedad de contextos requieren una exploración constante de las demandas sociales; lo que significa que al diseñar los perfiles profesionales y académicos deben tenerse en cuenta las opiniones de los diferentes grupos sociales y deben consultarse los organismos profesionales a nivel local, nacional e internacional. Por esto, al desarrollar el Proyecto Tuning, para definir las competencias genéricas y las competencias específicas de las diversas áreas temáticas, se recabó la opinión de los tres colectivos considerados como fundamentales ( los académicos universitarios, los empleadores y los graduados ), seleccionando, por lo que hace a las competencias genéricas, 10 competencias instrumentales (habilidades cognitivas, capacidades metodológicas para manipular el entorno, destrezas tecnológicas y destrezas lingüísticas), 8 competencias interpersonales (capacidades que facilitan los procesos de interacción social y de cooperación) y 12 competencias sistémicas o integradoras (concernientes a los sistemas como totalidad). El análisis comparativo de los resultados demostró que había muchas más coinci- dencias entre las opiniones de los empleadores y de los graduados que entre las opiniones de estos dos grupos y las de los académicos. En general, todas las destrezas interpersonales tienden a ser consideradas menos importantes por los académicos que por los graduados y empleadores.

En el caso de la titulación de Medicina, se han publicado numerosos listados de competencias; entre los que cabe señalar dos de carácter internacional (de la WFME y del IIME) y varios de ámbito nacional. Es de destacar que todos ellos incluyen gran parte de las competencias genéricas analizadas en el Proyecto Tuning; las cuales también han sido incluidas en la guía de ANECA para el diseño de planes de estudio dentro del Programa de Convergencia Europea.

Pero no basta con definir las competencias; hay que determinar que métodos se utilizarán para que los estudiantes las desarrollen y para evaluar el nivel de su adquisición a lo largo del currículo. En este sentido, cabe destacar el trabajo "Las competencias en el nuevo Paradigma Educativo para Europa" de la Universidad de Granada (2003), en el que, después de definir conceptualmente cada una de las competencias genéricas, se ofrecen unas directrices básicas sobre los métodos y técnicas de aprendizaje y de evaluación que pueden utilizarse (<http://www.upf.edu/ bolonya/obolonya/canvis/nouparad/docum/docencia.htm $>$ ). Por otra parte, es necesario determinar que unidades académicas serán responsables de la adquisición y evaluación de dichas competencias. Las competencias genéricas se adquieren normalmente a través de varias unidades; por lo que no pueden ligarse únicamente a una de ellas. Es necesario identificar las unidades que quedan implicadas en el aprendizaje de cada competencia para asegurar su evaluación y para garantizar que se adquieren los estándares adecuados de calidad. A título de ejemplo, son interesantes los programas como el de Ciencias Médicas de la Universidad de Indiana que detallan la participación de las diversas unidades académicas en los procesos de adquisición y evaluación de las competencias genéricas (http://medisci.indiana.edu/ Med Sci/comp.html)

\section{El proyecto ANECA y la convergencia de la titulación de Odontología en España al Proceso de Bolonia M.C. Martínez Álvarez \\ Vicedecana de Grado. Facultad de Odontología. Universidad Complutense de Madrid.}

La educación superior en Europa está inmersa en un proceso de modificación intensa encaminado a conseguir que la enseñanza de las mismas titulaciones, impartidas en los distintos estados que la forman, se armonice de manera que la total movilidad de estudiantes y profesionales pueda ser una realidad en un futuro cercano.

Este proceso de convergencia, iniciado en Bolonia en 1999, involucra una serie de elementos fundamentales como la generalización del sistema de créditos europeo, la nueva estructuración de titula- 
ciones y ciclos, la adopción del suplemento al diploma, el fomento de la movilidad de profesores y alumnos y el impulso de una cultura de calidad en la enseñanza superior. España se subió al tren de la convergencia europea en la Ley Orgánica de Universidades, marcando las líneas para posibilitar la nueva estructuración. Se creó la ANECA (Agencia Nacional de Evaluación de la Calidad y Acreditación) que, a través del Programa de Convergencia Europea, dio los primeros pasos para iniciar el proceso de cambio. Uno de los objetivos de este Programa fue crear el marco para que las universidades desarrollaran las bases de los futuros planes de estudio de sus titulaciones. Para conseguirlo, lanzó la Convocatoria de Ayudas para el Diseño de Planes de Estudio y Títulos de Grado. El proyecto presentado por el conjunto de todas las facultades de Odontología de España resultó financiable en la segunda de estas convocatorias y, así, se constituyó una red en donde las once facultades públicas y las cuatro privadas existentes en España empezaron a trabajar, bajo la coordinación del Profesor Mariano Sanz (actual Decano de la Faculta de Odontología de la Universidad Complutense de Madrid) y del Comité de Calidad de ese Centro, en el documento titulado: "Título de Grado en Odontología, que vio la luz en Junio de 2004.

Siguiendo la estructura del proyecto marcada por la ANECA, analizamos, en primer lugar, la situación de los estudios de Odontología en otros países de la Unión Europea a través de un cuestionario enviado a personas clave de la enseñanza odontológica en estos países. Seleccionamos luego, como los más adecuados al proceso de convergencia, los tres planes de estudios europeos que tomaríamos como base de nuestro diseño curricular. Investigamos la situación de oferta y demanda de acceso de estudiantes a nuestras facultades y de inserción laboral de los titulados de los últimos cinco años de las facultades de odontología españolas. A partir de múltiples documentos elaborados por organizaciones de la Unión Europea competentes en la materia, definimos el perfil profesional del odontólogo que queremos formar y los objetivos de la titulación. Una gran parte de nuestra actividad fue dedicada a determinar las competencias que este odontólogo debe haber adquirido al finalizar sus estudios de grado. Basados en el borrador del documento de competencias que, bajo la dirección de la Asociación de Educación Dental Europea, se estaba elaborando en ese momento, consensuamos las que pensamos que el odontólogo español ha de poseer. Una vez contrastadas por los colegios profesionales, sociedades científicas, alumnos de la licenciatura y profesores de universidad, vienen recogidas en el documento que presentamos. Listamos los contenidos formativos comunes obligatorios e instrumentales obligatorios que un estudiante de odontología debe adquirir para desarrollar las competencias antedichas, a la vez que propusimos el porcentaje de éstos y de los contenidos propios de cada universidad sobre el total del título. Por fin, organizamos siete bloques con estos contenidos, clasificando las enseñanzas de nuestra titulación como el proyecto ANECA determinaba, a los que asignamos, consensuadamente, los correspondientes créditos europeos.

El documento que recoge todos estos aspectos está actualmente siendo evaluado por la ANECA. Si es aprobado, será la base del Libro Blanco de la Odontología que esta Agencia presentará al Ministerio de Educación para la elaboración de las directrices propias de la titulación. Esperamos su pronta aprobación que permita a las distintas facultades de odontología de España iniciar la estimulante tarea de crear los planes de estudios que hagan de nuestros estudiantes unos profesionales para Europa.

\section{La Construcción del EEES en el ámbito de las Ciencias de la Salud: algunos elementos de debate en el marco de la complejidad M. Viader Junyent.}

Facultat de Psicologia, Universitat de Barcelona

El proceso de construcción del espacio europeo de educación superior (EEES) se estructura en torno a un conjunto de temáticas identificables pero al propio tiempo fuertemente inter-relacionadas, de forma que un abordaje profundo del tema exige la exploración de las interacciones entre los distintos aspectos

En el ámbito de las Ciencias de la Salud confluyen además algunos factores suplementarios a considerar. Debe citarse en este punto la presencia de aspectos normativos (en particular la existencia de directivas europeas), o el fuerte impacto social de las profesiones asociadas con estos estudios, relacionado en buena parte con la presencia del aspecto asistencial. Este último hecho implica un buen nivel de formación técnica y práctica, incluyendo obviamente la existencia de formación especializada complementaria para el ejercicio profesional en determinadas áreas, pero es perceptible también la necesidad de incidir en otras parcelas, relacionadas por ejemplo con las habilidades interpersonales. El impacto del perfil asistencial no debe hacer olvidar la presencia de un componente fundamental de ciencia básica y la importancia crucial de la investigación, ni la presencia en algunos casos del aspecto industrial, con la consiguiente necesidad de perfiles y respuestas diferenciados.

Explorando algunas de las particularidades del campo de las ciencias de la salud pueden citarse algunas cuestiones de interés en relación con el espacio europeo de educación superior.

. La posibilidad de una reflexión sobre el tema de las directivas europeas. Por su formulación parece claro que no han aportado una convergencia real en muchos aspectos importantes, aun considerando el necesario establecimiento de mínimos formativos. Es evidente la existencia de diferencias significativas en la formación recibida por los titulados en ciencias de la salud en distintos países europeos. Se pueden detectar fácilmente diferencias substanciales en cuanto a metodologías docentes (incluyendo la evaluación de aprendizajes), recursos humanos y materiales, características y eficacia del entrenamiento clínico, producción científica, en los criterios de calidad utilizados en la evaluación de las propias titulaciones, etc. Algunos estudios (realizados por ejemplo en el ámbito de la Odontología, pero posiblemente extrapolables) muestran considerables divergencias en la interpretación de la propia directiva europea, en la cantidad de tiempo dedicada a los distintos tópicos, en las condiciones de realización y exigencias de la formación práctica, etc. El proceso de construcción del EEES podría aportar algunos elementos de aproximación a través de sus instrumentos básicos: un sistema de titulaciones comparable, un sistema de créditos común, un posible catálogo de competencias compartido, un marco común en la evaluación de la calidad, etc. Ahora bien, será necesario un análisis cuidadoso de cada una de estas cuestiones: por citar un ejemplo, deberá valorarse cómo se enfoca la contabilización de horas de formación a partir del crédito europeo.

. La necesidad permanente de análisis de los perfiles profesionales asociados a las distintas titulaciones. Estos perfiles están sujetos a un proceso de cambio y diversificación más o menos acelerado a partir del planteamiento de nuevas demandas, de la evolución de las propias profesiones y del conocimiento disponible. En todo caso no debe confundirse la ampliación de 
ámbitos de aplicación con la definición de nuevos perfiles profesionales. En conjunto todo esto implica también la necesidad de adaptación progresiva de los programas formativos a las nuevas necesidades detectadas. Aquí será necesario valorar de forma adecuada el papel de cada uno de los niveles formativos (grado, master oficial, títulos de postgrado no oficiales, formación sanitaria especializada, doctorado). En todo caso no debe perderse de vista la necesidad de un perfil científico básico que permita un desarrollo potente de la investigación.

. La importancia de una reflexión amplia sobre el sentido del nuevo nivel de Master oficial. Es interesante valorar como punto de partida la situación actual de los estudios de postgrado en el campo de las ciencias de la salud, al margen de los estudios relativos a la formación sanitaria especializada: podemos constatar la existencia de una oferta amplia, a veces con carga en créditos importante (más de 150-200 créditos en algunos casos), dirigida muchas veces a la profundización temática con fuertes niveles de especialización, pero también a cuestiones relativas a gestión y organización de centros, peritaje, seguridad y prevención, etc. En este tema pueden identificarse algunas claves importantes de cara al futuro: por ejemplo, el interés de considerar el diseño e implantación de estudios de postgrado que, entre otras finalidades, permitan concretar y desarrollar los considerables elementos de transversalidad que se producen en el ámbito de las CC. de la salud, y también las confluencias con otras áreas temáticas (puede pensarse en ejemplos como Gerontología, Educación para la Salud, y otras). Por otra parte, parece conveniente una reflexión sobre cómo articular la distinción entre el Master oficial y los títulos no oficiales, ofrecidos en muchos casos por las propias universidades. ¿Es posible hablar, en relación con ello, de niveles de especialización diferentes?.

De manera relacionada, debe analizarse también el papel de la universidad en el impulso de nuevas ofertas formativas destinadas al reciclaje y mejora profesional.

. El planteamiento en profundidad del concepto de aprendizaje basado en competencias implica una revisión profunda de las metodologías docentes. De aquí se derivan muchas cuestiones de fondo como serían, por ejemplo, la redefinición del propio concepto de dedicación del profesorado y su contabilización en relación con su función docente, o la necesidad de establecer una diferenciación clara entre grado y postgrado en términos competencias adquiridas, en la línea de los descriptores de Dublín u otras aportaciones. Un ejemplo muy relevante en relación con las metodologías docentes es el del aprendizaje basado en problemas, una modalidad de docencia-aprendizaje que frecuentemente se plantea como alternativa preferente en el campo de las ciencias de la salud, y cuyas posibilidades y límites deberían ser explorados a fondo. Debe tenerse presente la coexistencia actual de proyectos innovadores importantes con sistemas docentes muy tradicionales.

Debe resaltarse la importante labor que vienen desarrollando distintas redes establecidas en el ámbito de las ciencias de la salud. Además de algunas redes europeas de interés (por ejemplo, redes temáticas Erasmus, redes vinculadas al proyecto Tuning, etc.) en nuestro sistema universitario se ha producido una reflexión importante en el marco de los proyectos de diseño de titulaciones articulados en torno a las convocatorias ANECA. Al margen de la valoración concreta que pueda realizarse sobre los resultados o las propias convocatorias, el trabajo de estas redes ofrece elementos de reflexión y debate que no deberían ser desaprovechados.

\section{Espacio de educación superior europeo J. de Nadal i Caparà}

Director del Institut d'Estudis de la Salut.

Generalitat de Catalunya

En el contexto de la convergencia europea de los estudios en ciencias de la salud y en coherencia con las aportaciones de otros miembros de la Mesa, creo que pueden tener interés los proyectos de evaluación de la competencia en el ámbito de los profesionales sanitarios que viene realizando el Institut d'Estudis de la Salut des del año 1994.

La calidad de los servicios está vinculada tanto a una buena planificación y organización de los recursos económicos y en infraestructuras, como a la competencia de sus profesionales.

El sistema sanitario, como institución que presta servicios, hace un uso intensivo de recursos humanos altamente cualificados.

Por tanto, la definición de las competencias de los profesionales sanitarios y la utilización de métodos de evaluación de estas competencias, son elementos clave para garantizar la calidad de los servicios sanitarios que se prestan.

El IES, mediante el Consell Català d'Especialitats en Ciències de la Salut, publicó un documento sobre las competencias de diferentes profesionales sanitarios que sirvió de base para los proyectos de evaluación de la competencia profesional.

También ha colaborado con las facultades de medicina en la definición de las competencias de los estudiantes de medicina. Ha colaborado con la Federación Mundial de Educación Médica en la elaboración y publicación en lengua catalana de los estándares globales para la mejora de la calidad en la educación médica básica.

\section{NIVELES DE EVALUACIÓN}

En el desarrollo profesional continuado individual se pueden distinguir tres niveles diferentes de evaluación: al finalizar la formación pregraduada, al finalizar la formación postgraduada con propósitos de certificación de la especialidad y durante el ejercicio profesional con propósitos de recertificación y carrera profesional.

Es necesario, por tanto, dirigir acciones evaluadoras para estos tres niveles que serán necesariamente diferentes, no sólo en cuanto a contenidos sino también en cuanto a métodos a utilizar. Es necesario también utilizar los métodos de evaluación objetiva para la selección de los profesionales para adecuarlos en su lugar de trabajo.

En el año 1994 se realizó el primer proyecto de evaluación de la competencia y hasta la actualidad se han realizado más de 150 proyectos y se ha evaluado a más de 12.000 profesionales.

Estos proyectos se han desarrollado en colaboración, en el ámbito de pregrado, con las facultades de medicina y las escuelas universitarias de enfermería, en el ámbito de postgrado con los responsables de la formación de residentes de la especialidad de medicina familiar y comunitaria y en el ámbito del ejercicio profesional con propósitos de certificación profesional, con las sociedades científicas y los colegios profesionales.

\section{MÉTODOS DE EVALUACIÓN}

Los métodos utilizados para evaluar las competencias, se basan fundamentalmente en las simulaciones que intentan reproducir situaciones similares de la vida real en condiciones estandarizadas, que permiten que los observadores puedan analizar las actuaciones específicas que se pretenden evaluar.

Una mención especial es la prueba conocida como OSCE 
(Objective Structures Clinical Examination) o Evaluación Clínica Objetiva y Estructurada (ACOE).

La ACOE es un formato de prueba en el que se pueden englobar diferentes métodos evaluativos. El formato básico consiste en que los candidatos efectúan una rotación por un circuito secuencial de estaciones en las que se les pide realizar una variedad de diferentes habilidades. En muchas de estas estaciones se utilizan pacientes simulados estandarizados, casos por ordenador, maniquíes, equipamiento médico real, análisis de pruebas complementarias (ECG, RX, analítica, etc.), entre otros.

Respecto a los proyectos de valoración formativa de los residentes médicos especialistas en formación además de dichas simulaciones también se han utilizado el portafolio o cuaderno de autoaprendizaje.

El portafolio es un acopio de la evidencia del autoaprendizaje o de consecución de objetivos, para una finalidad específica. Habitualmente se trata de material impreso aunque puede incluir cualquier otro como por ejemplo videograbaciones de la práctica profesional.

Este método de evaluación se puede utilizar tanto en la formación de pregrado y de postgrado como instrumento de valoración formativa o evaluación sumativa, como durante el ejercicio profesional para la recertificación de los profesionales sanitarios.

\section{Situación actual de la convergencia europea de los estudios de Ciencias de la Salud J. Sánchez Carralero} Delegat del Rector per Convergència Europea

La Universitat de Barcelona contempla el EEES como una gran oportunidad de estimular un proceso de "aggiornamento" de la actividad académica $\mathrm{y}$, desde hace tiempo, lo tiene definido como una línea estratégica de actuación, con lo que esto comporta de focalización de esfuerzos y recursos.

En un primer momento la actividad consistió fundamentalmente en informar, sobre todo al profesorado, puesto que tenía que ser el protagonista del cambio. Hasta el presente podemos decir que se han desarrollado varios tipos de sesiones informativas, por centros, colectivos, etc., que permiten estar seguros de que, prácticamente, toda la comunidad está enterada de las líneas generales del proceso.
Con respecto a cuestiones más concretas, podemos distinguir grandes líneas de actuación:

1. Formación del profesorado, con líneas especificas de trabajo. Prácticamente se está haciendo una formación a demanda de los centros.

2. Desarrollo de una política de planes docentes, que va desde la conceptualización de los diferentes apartados que constituyen los planes docentes: recursos humanos o materiales, hasta la elaboración de las guías docentes.

a. Diseño de instrumentos específicos: GRAD (Gestión de recursos docentes) y aplicativo de las guías docentes

3. Potenciación de herramientas que faciliten la puesta en marcha de las nuevas funciones docentes del profesorado: campus virtual avanzado, CRAl.

4. Producción de documentación de apoyo

5. Puesta en marcha de pruebas piloto:

a) Las titulaciones piloto UB, la misión de los cuales fue empezar el proceso de reflexión en los diferentes ámbitos académicos.

b) Las pruebas piloto *DURSI, que implican la transformación académica de cursos completos

a) Las pruebas piloto de postgrados. Los nuevos según ciclos del EEES suponen una aproximación académica novedosa respecto de la situación actual. En la UB creemos, como otras muchas universidades de todas partes, que el éxito de la oferta de esta docencia marcará los rasgos diferenciales entre las instituciones. Es por esta razón que aún con la incertidumbre del marco legislativo resulta conveniente hacer una primera oferta de postgrados. Para ello se ha pedido a los diversos centros cuáles son las propuestas que están en condiciones de llevar a término de cara al curso 2005-06

Naturalmente quedan otras muchas acciones a desarrollar en el contexto del EEES, como por ejemplo los nuevos roles de los estudiantes en su proceso de aprendizaje, la información al PAS, etc. Por otro lado, el desarrollo del marco legislativo irá perfilando las diversas acciones ya en curso.

En cualquier caso, conviene tener muy presente que todo este cambio se producirá, en nuestra institución, sobre una importante base de tradición en la innovación docente, lo que se traduce en que, aún teniendo en cuenta las dificultades, nuestras probabilidades de éxito son grandes. 